\title{
COMPLETE DISTRIBUTIVITY AND ORDERED GROUP LATTICES
}

\author{
CECELIA LAURIE ${ }^{1}$
}

\begin{abstract}
Arveson has recently generalized an important result of Andersen's about continuous nests to a larger class of lattices. Andersen's result is a base for much of the recent interesting work on compact perturbations and similarity of nest algebras. This paper investigates further the structure of such lattices. It is shown that the ordered group lattices with $\Sigma$-continuous measures introduced by Arveson are completely distributive. This immediately implies various nice properties of $\operatorname{Alg} \mathscr{L}$, the associated algebra of operators leaving such a lattice $\mathscr{L}$ invariant. (Among these are the fact that the rank one operators in $\mathrm{Alg} \mathscr{L}$ are dense in $\mathrm{Alg} \mathscr{L}$ and that $\mathrm{Alg} \mathscr{L}+\mathscr{K}$ is norm closed where $\mathscr{K}$ denotes the compact operators.)
\end{abstract}

Recently much interesting work has been done on similarity of nest algebras and compact perturbations of nest algebras $[1,2,4,10,9]$. This work is based on a result of Andersen [1, Theorem 3.5.5] concerning continuous nests. This result has been generalized by Arveson [3]. The setting for this generalization involves a new class of commutative subspace lattices, which we will call ordered group lattices. There is a natural desire to explore further properties of such a lattice $\mathscr{L}$ and of $\mathrm{Alg} \mathscr{L}$, the associated algebra of operators leaving $\mathscr{L}$ invariant, with the eventual hope of extending similarity and compact perturbation results. Some ingredients in these results for nests are the facts that if $\mathscr{N}$ is a nest, then $\mathrm{Alg} \mathscr{N}+\mathscr{K}$ is closed (where $\mathscr{K}$ is the algebra of compact operators) and Alg $\mathscr{N}$ is hyperreflexive. The main result of this paper is that the ordered group lattices derived from $\Sigma$-continuous projection valued measures are completely distributive. Completely distributive (commutative) lattices are of interest because their associated algebras have many nice properties. In particular, for such a lattice $\mathscr{L}$, we have that the subalgebra generated by the rank-one operators in $\mathrm{Alg} \mathscr{L}$ is dense in $\mathrm{Alg} \mathscr{L}$ and that $\mathrm{Alg} \mathscr{L}+\mathscr{K}$ is closed.

Throughout this paper all Hilbert spaces are separable. A subspace lattice is a lattice of projections acting on a (separable) Hilbert space which contains zero and the identity and which is closed in the strong operator topology. A subspace lattice $\mathscr{L}$ is commutative if the projections in $\mathscr{L}$ commute with each other. A CSL algebra is a reflexive algebra whose subspace lattice is commutative. A lattice is completely distributive if it permits distribution of the lattice operations over families of arbitrary cardinality. We refer the reader to [6 or 8] for a precise definition and a discussion of equivalent characterizations. The characterization of complete distributivity for subspace lattices that we will use is due to Longstaff [12]. We first need a

Received by the editors August 7, 1984 and, in revised form, October 31, 1984.

1980 Mathematics Subject Classification. Primary 47D25.

${ }^{1}$ Partially supported by a grant from the Research Grants Committee of the University of Alabama. 
couple of definitions. For $M$ and $L \in \mathscr{L}$, define

$$
M_{-}=\vee\{N \in \mathscr{L} \mid M \nless N\} \quad \text { and } \quad L_{*}=\wedge\left\{M_{-} \mid M \in \mathscr{L}, M \nless L\right\} .
$$

A subspace lattice is completely distributive iff $L_{*}=L$ for all $L \in \mathscr{L}$ [12].

Let $G$ be a second countable locally compact abelian group and let $\Sigma$ be a cone in $G$, i.e. $\Sigma$ satisfies

(i) $\Sigma \cap-\Sigma=\{0\}$,

(ii) $\Sigma+\Sigma \subseteq \Sigma$,

(iii) $\Sigma$ is the closure of its interior.

We define a partial order $\leqslant$ in $G$ by $x \leqslant y$ if $y-x \in \Sigma$. We say that $(G, \Sigma)$ is an ordered group. A Borel set $E \subseteq G$ is called increasing if, for every $x \in E$ and $y \in G$, $x \leqslant y$ implies $y \in E$. Let $m$ be a $\sigma$-finite measure on $G$. Let $P_{E}$ denote the projection on $L^{2}(G, m)$ given by multiplication by $\chi_{E}$. We define

$$
\mathscr{L}(\Sigma, m)=\left\{P_{E}: E \text { increasing }\right\} \text {. }
$$

We will call this an ordered group lattice. We say that $m$ is $\Sigma$-continuous if the function $x \in G \mapsto m(E+x)$ is continuous for every increasing set $E$. These lattices and the notion of $\Sigma$-continuity of a measure were introduced by Arveson in [3]. There he shows that $\mathscr{L}(\Sigma, m)$ is a commutative subspace lattice and that the $\Sigma$-continuity of a measure $m$ implies that $m(\partial E)=0$ for all increasing sets $E$ and in fact is equivalent to $m(\partial E)=0$ for all closed increasing sets $E$. (Here $\partial E$ denotes the boundary of $E$.)

We will now show that, for $(G, \leqslant, m)$ with $\Sigma$-continuous $m$, the lattice $\mathscr{L}(\Sigma, m)$ is completely distributive. We first require some technical lemmas and definitions. Define the support of a measure $m$ to be

$$
\text { supp } m=\{x \in G \mid m(U) \neq 0 \text { for every open set } U \text { containing } x\} \text {. }
$$

For $x \in G$, let $\hat{I}_{x}=\{z \in G \mid x \leqslant z\}$ and $\hat{D}_{x}=\{z \in G \mid z \leqslant x\}$. Note that $\hat{I}_{x}=x+$ $\Sigma$ and $\hat{D}_{x}=x-\Sigma$. The corresponding projection determined by multiplication by the characteristic function of $\hat{I}_{x}$ (respectively $\hat{D}_{x}$ ) will be denoted by $I_{x}$ (resp. $D_{x}$ ). If $E$ is a subset of $G, \bar{E}$ will denote the closure of $E$.

Lemma 1 [6, Lemma 6]. For every $x \in G$,

$$
D_{x} \wedge\left(I_{x}\right)_{-}=0
$$

LemMa 2. Let $\mathscr{L}(\Sigma, m)$ be an ordered group lattice such that $m$ is $\Sigma$-continuous. Let $S$ be a Borel set such that $m(S)>0$. Then there exists $x \in S$ and $z \in$ int $\hat{I}_{x}=x+$ int $\Sigma$ such that $m\left(\hat{I}_{x} \cap S\right)>0$ and $m\left(\hat{I}_{z} \cap S\right)>0$.

Proof. Suppose that $m\left(\hat{I}_{x} \cap S\right)=0$ for all $x$ in $S$. Let $\left\{x_{i}\right\}$ be a countable dense subset of $S$ and define $E=\bigcup_{i=1}^{\infty} \hat{I}_{x_{i}}$. We have that $E$ is increasing and $m(E \cap S) \leqslant$

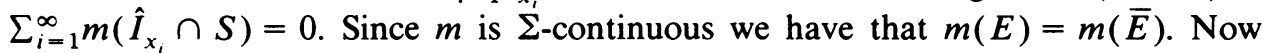
$S \subseteq\left\{\bar{x}_{i}\right\} \subseteq \bar{E}$ so $m(S)=m(\bar{E} \cap S)=m(E \cap S)=0$, contradicting the assumption that $m(S)>0$. Hence there exists an $x \in S$ such that $m\left(\hat{I}_{x} \cap S\right)>0$.

To find the desired $z$, first choose a sequence $\left\{\sigma_{n}\right\}$ such that $\sigma_{n} \in$ int $\Sigma, \sigma_{n+1} \leqslant \sigma_{n}$, and $\lim _{n \rightarrow \infty} \sigma_{n}=0$. (To see that such a sequence exists, see [3, Proof of Proposition 
6.2].) Let $x$ be such that $m\left(\hat{I}_{x} \cap S\right)>0$. Using the facts that $\Sigma$ is an increasing set and that $m$ is $\Sigma$-continuous, we have that

$$
\lim _{n \rightarrow \infty} m\left(\hat{I}_{x+\sigma_{n}}\right)=\lim _{n \rightarrow \infty} m\left(\Sigma+\left(x+\sigma_{n}\right)\right)=m(\Sigma+x)=m\left(\hat{I}_{x}\right)
$$

and hence that $\lim _{n \rightarrow \infty} m\left(\hat{I}_{x+\sigma_{n}} \cap S\right)=m\left(\hat{I}_{x} \cap S\right)$ since $\hat{I}_{x+\sigma_{n}}$ is an increasing sequence of sets.

Since $m\left(\hat{I}_{x} \cap S\right)>0$ we can conclude that there exists an $N$ such that, for $z=x+\sigma_{N} \in x+$ int $\Sigma$, we have $m\left(\hat{I}_{z} \cap S\right)>0$.

THEOREM 3. If $\mathscr{L}=\mathscr{L}(\Sigma, m)$ is an ordered group lattice such that $m$ is $\Sigma$-continuous, then $\mathscr{L}$ is completely distributive.

Proof. Suppose $\mathscr{L}$ is not completely distributive, i.e. there exists an $L \in \mathscr{L}$ such that $L_{*} \neq L$. Let $\hat{L}$ (respectively $\hat{L}_{*}$ ) be an increasing set such that the projection determined by $\hat{L}$ (resp. $\hat{L}_{*}$ ) equals $L$ (resp. $L_{*}$ ). Let $R$ be the closed set $\hat{L}_{*} \cap$ (int $\left.\hat{L}\right)^{c}$. Note that, since $m(\partial \hat{L})=0$ and $m\left(\partial \hat{L}_{*}\right)=0$, we have that $m(R)=m\left(\hat{L}_{*} \cap \hat{L}^{c}\right)$. Thus $m(R)>0$ by assumption. Let $S=\left.\operatorname{supp} m\right|_{R}$. Since $R$ is closed, $S \subseteq R$ and thus we have $m(S)=m(R)=m\left(\hat{L}_{*} \cap \hat{L}^{c}\right)$. Since $m(S)>0$, by Lemma 2 we can choose $x \in S$ and $z \in x+$ int $\Sigma$ such that $m\left(\hat{I}_{x} \cap S\right)>0$ and $m\left(\hat{I}_{z} \cap S\right)>0$. We thus have

(i) $m\left(\hat{I}_{z} \cap \hat{L}_{*} \cap \hat{L}^{c}\right)>0$.

Next note that $x \in$ int $\hat{D}_{z}=z-$ int $\Sigma$. Since $x \in S=\left.\operatorname{supp} m\right|_{R}$ we have that $m$ (int $\left.\hat{D}_{z} \cap R\right)>0$. Thus we can conclude that

(ii) $m$ (int $\left.\hat{D}_{z} \cap \hat{L}_{*} \cap \hat{L}^{c}\right)>0$.

Statement (i) implies that $I_{z} \nless L$ and hence that $L_{*} \leqslant\left(I_{z}\right)_{-}$(by definition of $L_{*}$ ). Let $\left(\hat{I}_{z}\right)_{-}$be an increasing set such that the corresponding projection determined by mutiplication by the characteristic function of $\left(\hat{I}_{z}\right)_{-}$equals $\left(I_{z}\right)_{-}$. Lemma 1 implies that int $\hat{D}_{z} \subseteq\left(\hat{I}_{z}\right)_{-}^{c}$. Thus statement (ii) implies that $m\left(\hat{L}_{*} \cap\left(\hat{I}_{z}\right)_{-}^{c}\right)>0$ which is a contradiction of the previous conclusion that $L_{*} \leqslant\left(I_{z}\right)_{\text {. }}$. Thus $\mathscr{L}$ must be completely distributive.

A projection valued measure on $G$ is a countably additive function $P$ from the Borel sets of $G$ to the projections on a separable Hilbert space $\mathscr{H}_{P}$ satisfying $P(\varnothing)=0$ and $P(G)=I$. Such a $P$ is called $\Sigma$-continuous if $P(\partial E)=0$ for every closed increasing set $E$ [3]. Arveson's generalization of Andersen's theorem was formulated in terms of projection valued measures [3].

Corollary 4. Let $(G, \Sigma)$ be an ordered group and let $P$ be a $\Sigma$-continuous projection valued measure. Then

$$
\mathscr{L}=\{P(E): \text { E increasing }\}
$$

is a completely distributive subspace lattice of $\mathscr{H}_{P}$.

Proof. Let $\rho$ be a faithful normal state on $\mathscr{L}\left(\mathscr{H}_{P}\right)$ and let $m$ be a probability measure on $G$ defined by $m(S)=\rho(P(S))$. We have that $\mathscr{L}(\Sigma, m)$ is an ordered group lattice with $\Sigma$-continuous measure. Following [3, Proof of Proposition 7.2], we see that there exists a strongly continuous lattice isomorphism $\theta$ from $\mathscr{L}(\Sigma, m)$ onto 
$\mathscr{L}$. Since $\theta(M) \leqslant \theta(L)$ iff $L \leqslant M$ and since $\theta$ is strongly continuous, we have that $\theta\left(L_{*}\right)=\theta(L)_{*}$. Hence since $\mathscr{L}(\Sigma, m)$ is completely distributive we can conclude that $\mathscr{L}$ is completely distributive.

COROllary 5. Let $(G, \Sigma)$ be an ordered group. Let $\mathscr{L}=\{P(E)$ : $E$ increasing $\}$ where $P$ is a $\Sigma$-continuous projection valued measure. Then $\mathscr{L}$ has the following properties:

6.1. The rank one operators in $\mathrm{Alg} \mathscr{L}$ are dense in $\mathrm{Alg} \mathscr{L}$ (in any of the ultraweak, ultrastrong, weak, or strong topologies).

6.2. Alg $\mathscr{L}+\mathscr{K}$ is norm closed where $\mathscr{K}$ denotes the compact operators on $\mathscr{H}_{P}$.

6.3. If $\mathscr{L}_{2}$ is any other subspace lattice of $\mathscr{H}_{P}$, then $\mathrm{Alg} \mathscr{L} \otimes \operatorname{Alg} \mathscr{L}_{2}=\operatorname{Alg}\left(\mathscr{L} \otimes \mathscr{L}_{1}\right)$ where $\bar{\otimes}$ denotes the ultraweakly closed algebra generated by elementary tensors and $\otimes$ denotes the subspace lattice generated by elementary tensors.

Proof. Statement 6.1 follows from Corollary 4 and Theorem 3 in [11]. Statement 6.2 follows from Statement 6.1 and Theorem 1.1. in [5]. Statement 6.3 follows from Corollary 4 and Theorem 2.2 in [7].

REMARK. The remark near the end of Arveson's paper [3] that Alg $\mathscr{L}+\mathscr{K}$ is norm-closed for $\mathscr{L}$ as in Corollary 5 is not stated completely correctly. This result was known to Arveson only for the case $\mathscr{L}=\mathscr{L}(\Sigma, m)$ where $m$ is Haar measure on $G$.

The author would like to thank Bob Moore and Alan Hopenwasser for several helpful conversations.

The author was partially supported by a grant from the Research Grants Committee of the University of Alabama.

\section{REFERENCES}

1. N. T. Anderson, Compact perturbations of reflexive algebras, J. Funct. Anal. 38 (1980), 366-400.

2. Similarity of continuous nests, Bull. London Math. Soc. 15 (1983), 131-132.

3. W. Arveson, Perturbation theory for groups and lattices, J. Funct. Anal. 53 (1983), 22-73.

4. K. R. Davidson, Similarity and compact perturbations of nest algebras, J. Reine Angew. Math. 348 (1984), 72-87.

5. T. Fall, W. Arveson and P. Muhly, Perturbations of nest algebras, J. Operator Theory 1 (1979), 137-150.

6. A. Hopenwasser, C. Laurie and R. Moore, Reflexive algebras with completely distributive subspace lattices, J. Operator Theory 11 (1984), 91-108.

7. J. Kraus, Tensor products of reflexive algebras, J. London Math. Soc. (2) 28 (1983), 350-358.

8. M. S. Lambrou, Completely distributive lattices, Fund. Math. 119 (1983), 227-240.

9. E. C. Lance, Cohomology and perturbations of nest algebras, Proc. London Math. Soc. (3) 43 (1981), $334-356$.

10. D. R. Larson, Nest algehras and similarity transforms, Ann. of Math. (to appear)

11. C. Laurie and W. E. Longstaff, A note on rank-one operators in reflexive algebras.,Proc. Amer. Math. Soc. 89 (1983). 293-297.

12. W. E. Longstaff, Strongly reflexile lattices, J. London Math. Soc. (2) 11 (1975), 491-498.

Dipartilent of Mathematics, University of Alabama, Univirsity, Al.abama 35486 